\title{
BMJ Open Should both iodised and non-iodised salt be made available in Chinese cities? A cross-sectional survey
}

\author{
J Yu, ${ }^{1}$ P Liu, ${ }^{2}$ Y Liu, ${ }^{2}$ S J Liu, ${ }^{2}$ D J Sun ${ }^{3}$
}

To cite: Yu J, Liu P, Liu Y, et al. Should both iodised and non-iodised salt be made available in Chinese cities? A cross-sectional survey. BMJ Open 2014;4:e005397. doi:10.1136/bmjopen-2014005397

- Prepublication history for this paper is available online To view these files please visit the journal online (http://dx.doi.org/10.1136/ bmjopen-2014-005397)

YJ and LP contributed equally.

Received 4 April 2014

Revised 18 June 2014 Accepted 23 June 2014

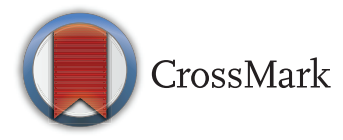

For numbered affiliations see end of article.

Correspondence to Dr Sun Dianjun; hrbmusdj@163.com

\section{ABSTRACT}

Objective: To contribute evidence relevant to the policy of supplying iodised salt (IS), non-iodised salt (NIS) or both in Chinese cities.

Design: Subnational telephone interview survey. Setting: China.

Participants: Totally, 24557 telephone numbers were dialled and 4833 citizens accepted the telephone interview. The telephone numbers were randomly selected by random digit dialling and a MitofskyWaksberg two-stage sampling method in 17 capital cities and 6 coastal cities from 17 iodine deficiency disorder (IDD)-eliminated provinces (municipalities).

Results: The 4833 citizens finished the telephone interview. Among them, 3738 (77.3\%) citizens chose IS, $481(10 \%)$ citizens chose NIS, and the others chose both IS and NIS. The citizens' awareness rates of IDD and IDD preventive measures were $68.7 \%$ and $62.5 \%$, respectively.

Conclusions: It is not a suitable time to supply IS and NIS simultaneously in the developed cities of China, but a pilot project may be conducted in the cities where IDD has been sustainably eliminated, there is strong awareness of IDD and the population can make informed decisions regarding IS. IDD health education should be further strengthened, especially regarding the potential for $I Q$ damage.

\section{INTRODUCTION}

Approximately $20 \%$ of the total global population resides in China, which has one of the most severe iodine deficiency epidemics, in part due to the external environment. Iodine deficiency disorders (IDD) were once widespread and caused great damages. Universal salt iodization (USI) has been adopted for IDD prevention and control and has proven to be an effective measure since 1994. By 2000, 17 provinces (municipalities) in China achieved the goal of eliminating IDD and have sustained this outcome for 10 years based on the WHO criteria for IDD elimination. ${ }^{1}$

However, an excess of iodine has been discovered, and new issues, ${ }^{2-4}$ including the increasing rate of thyroid disease and the

\section{Strengths and limitations of this study}

- The article initially describes the residents' preferences on salt type (iodised (IS) or non-iodised salt (NIS)) and their awareness of iodine deficiency disorders and its prevention measures.

- The telephone interview method is biased towards those residents with a fixed-line telephone. The pencil-and-paper interview method, rather than computer-aided telephone interviews, was used.

- Options for explaining the IS/NIS choice should have been more detailed.

elevated health awareness of the Chinese population, have started a debate about the ability of the population to make informed choices to use iodised salt (IS) or noniodised salt(NIS). As we know, some developed European countries, such as Belgium, Finland, France, Germany, Greece, Ireland, Italy and the Netherlands, supply IS and NIS simultaneously in the market. ${ }^{5}$ Residents of these countries can purchase IS or NIS voluntarily. In developed cities in provinces that have demonstrated the sustainable elimination of IDD, is it the right time to supply IS and NIS simultaneously? If so, can these populations remain free of IDD? Finally, is there a proper method to demarcate cities to which IS and NIS is supplied simultaneously or to which only IS is supplied? To answer these questions, the subjective preference of the residents about choosing IS or NIS and the relevant influencing factors should be examined.

Door-to-door interviewing is usually used to conduct such investigations. Considering the associated time and money expenditures, a cross-sectional telephone interview is a feasible alternative and is a widespread method that was adopted earlier and used by different countries all over the world. ${ }^{6}$ Successful cases of the use of cross-sectional telephone interviewing in China include KnowledgeAttitude-Practice (KAP) surveys on influenza, smoking and pollinosis. ${ }^{7-10}$ 
The application of cross-sectional telephone interviewing is based on educational and economic levels, in addition to the telephone popularisation rate. In China, by the end of 2009, the national average Urban Per Capita Disposable Income was 17175 RMBY, and some developed cities (municipalities) in the IDD-eliminated provinces were most at or beyond the national average (table 1). ${ }^{11}$ Additionally, the national fixed line penetration had increased to 314 million people (23.5 telephones/100 persons) and 212 million people in the cities (34.1 telephones/100 persons). These facts made telephone interview a representative and feasible way to research the residents' KAP towards IDD.

As far as we know, there were no such records about the residents' subjective preferences between IS and NIS, and no research on the residents' awareness of IDD in developed cities (municipalities) has been conducted in recent years. Hence, the National Centre for Endemic Disease Control (CEDC) launched this project in April 2010, with the aim of obtaining data on the above aspects to decide whether supplying IS and NIS simultaneously is feasible in developed cities in IDD-eliminated provinces. The methods and results may provide evidence or references for similar regions in the world.

\section{METHODS}

\section{City sites to be investigated}

The 17 capital cities of the 17 provinces ( 3 municipalities, 6 coastal provinces and 8 inland provinces) that had achieved the goal of IDD elimination in 2000 were selected (table 1). One coastal city was also selected in each of the above coastal provinces. A total of 23 cities were included in the study.

\section{Target population}

Citizens who had fixed telephones in the 23 selected cities.

\section{The main contents of the questionnaire}

- If IS and NIS are supplied simultaneously, which will you choose? And why?

- Have you heard of IDD? If so, what are the effects? How is IDD prevented?

- Can you tell us your occupation and age group?

\section{Sampling method and sampling size}

Random digit dialling and the Mitofsky-Waksberg twostage sampling method were used in this study. ${ }^{12}$ In each city, only the main urban zones were investigated, and two districts were selected randomly from each main urban zone. At least 200 effective samples were sampled in each city included in the survey. The sample quota was divided evenly between each district. The sample size was calculated according to formula 1 ; the positive response rate $(\pi)$ was approximately $70 \%$ according to the pilot survey and the allowable error $(\delta)$ was 0.5 .
Thus, the calculated sample size was 170 responses per city; therefore, 200 responses per city were considered adequate:

$$
\mathrm{n}=\left\{\frac{\mu_{\alpha}^{2} \times \pi(1-\pi)}{\delta}\right\}
$$

where $\pi$ is the positive rate and $\delta$ is the allowable error.

\section{Telephone number acquisition}

If the fixed telephone of the neighbourhood offices in the selected district was seven-digit or eight-digit numbers, the first three or four digits were deemed to be the direction code. The last four digits were obtained by the Random function (=Rand ()$\times 10000)$ in Microsoft Office Excel. If the function produced randomised numbers less than four digits, and ' 0 ' was supplemented in front of the generated numbers. The number of telephone numbers created randomly should be at least five times the effective sample size, that is, at least 1000 telephone numbers should be created per city.

\section{Quality control}

The research was approved by the review board of the Harbin Medical University. Participant consent was acquired when they answered the telephone. The survey programme and survey result database were designed and delivered to the project cities by the national CEDC. The survey programme was improved based on a pilot survey, and the questionnaire had clear questions that prompted simple answers and avoided providing hints to the residents. Sensitive questions, such as age, were designed as "Which age group do you belong to? $<20$, 20-30, 30-40...".

In the programme, the investigation was carried out in a unified manner, including the order and pattern of the questions, the dialling time $(18: 30-21: 00)$, and instructions for making notes of the answers and filling the database.

To avoid investigation and report biases and to improve the research participants' cooperation, the interviewers were trained on their attitude, mood and countermeasures in case of emergency by the project provinces themselves according to the rules of the survey programme. For example, some related questions put forward by residents could be answered only after the interview was finished to avoid bias.

To guarantee the reliability of the research information, a return visit was conducted after a project city finished the telephone interviews. The quality control officer of the provincial Centre for Disease Control (CDC) or CEDC visited the citizens who had accepted the interview. At least $10 \%$ of the respondents were randomly sampled to complete the return visit questionnaire. When the coincidence rate of survey information was greater than $80 \%$, the investigation results of the city 
$\underline{\text { Table } 1 \text { Provinces and cities surveyed }}$

\begin{tabular}{|c|c|c|c|c|c|c|c|}
\hline \multirow[b]{2}{*}{ Category } & \multirow[b]{2}{*}{ Provinces } & \multicolumn{3}{|c|}{ Provincial capital } & \multicolumn{3}{|c|}{ Coastal cities } \\
\hline & & Name & Telephone $^{*}$ & Income $e^{\star \star}$ & Name & Telephone* & Income $^{\star \star}$ \\
\hline \multirow[t]{6}{*}{ Coastal province } & Hebei & Shijiazhuang & - & 16607 & Tangshan & 24.58 & 18053 \\
\hline & Shandong & Ji'nan & 39.28 & 22272 & Yantai & 28.27 & 21125 \\
\hline & Jiangsu & Nanjing & 38.91 & 25504 & Nantong & 35.80 & 21001 \\
\hline & Zhejiang & Hangzhou & 48.23 & 26864 & Zhoushan & 53.45 & 24082 \\
\hline & Guangdong & Guangzhou & 61.28 & 27610 & Shenzhen & 52.64 & 29244 \\
\hline & Guangxi & Nanning & 16.61 & 16254 & Beihai & - & 15134 \\
\hline \multirow[t]{8}{*}{ Inland province } & Heilongjiang & Harbin & 21.66 & 15887 & & & \\
\hline & Jilin & Changchun & - & 16277 & & & \\
\hline & Shanxi & Taiyuan & 44.31 & 15607 & & & \\
\hline & He'nan & Zhengzhou & 29.28 & 17117 & & & \\
\hline & Anhui & Hefei & 32.47 & 17158 & & & \\
\hline & Hubei & Wuhan & 40.57 & 18385 & & & \\
\hline & Jiangxi & Nanchang & 32.37 & 16472 & & & \\
\hline & Hu'nan & Changsha & 33.28 & 20238 & & & \\
\hline \multirow[t]{3}{*}{ Municipalities } & Beijing & Beijing & 50.89 & 26738 & & & \\
\hline & Shanghai & & & & Shanghai & 48.70 & 28838 \\
\hline & Tianjin & & & & Tianjin & 31.36 & 21430 \\
\hline
\end{tabular}

can be regarded as qualified, and otherwise, the city should be reinvestigated.

\section{Definition of telephone number}

Success: respondent finished the entire questionnaire; Rejection: respondent refused to answer or did not finish the questionnaire; No answer: the phone was busy both the first time and when redialling after $5 \mathrm{~min}$; Invalid number: the phone number was vacant, fax, switchboard or phone quit.

\section{Data processing and statistical analysis}

Epi info 3.5.1 (CDC, Atlanta, Georgia, USA) was used for the database design, and SPSS V.17.0 was utilised for analysis. The level of significance was set at 0.05 .

\section{RESULTS}

\section{Response situation}

The flow chart is presented in figure 1 . The survey included 235 districts of 120 main urban zones in 23 cities. In total, 446 direction numbers were chosen, and 24557 telephone numbers were dialled, of which 13215 $(53.8 \%)$ were valid and 11342 were invalid numbers. An invalid number was a number that was not used as a telephone number. Among the 13215 valid telephone numbers, 4833 successfully finished the questionnaire, 1949 answered the phone but refused to answer the questions and 6433 telephones were not answered. The answered numbers (successes and rejections) were 6782 and accounted for $51.3 \%$ of the valid numbers. The total response rate $(4833 /(4833+1949) \times 100 \%)$ was $71.3 \%$ ( figure 2 ).

\section{Basic information about the interviewee}

The age and occupation questions were answered by 4861 and 4857 residents, respectively. The age and occupation proportion were similar between the capital city (including Beijing) and the coastal city (including Tianjin and Shanghai) residents. The residents' age were mainly distributed between 20 and 60 years, and this range accounted for $80 \%$ of the respondents. However, compared with the population composition from the ' 2009 China Health Statistical Yearbook' ${ }^{13}$ and the Chinese population pyramid, ${ }^{14}$ the $10-20$ age group had a smaller constituent ratio. This may lead to a nonrepresentative sample of the Chinese population, although this may be acceptable in this study because children rarely buy salt. The residents were evenly distributed in each profession (accounting for approximately $10 \%$ ), except for the professions of servicemen, students and irregular professions, which had small sample sizes.

\section{Proportion of respondents choosing IS or NIS}

Of the residents investigated, 4865 answered the question 'If IS and NIS are supplied simultaneously, which will you choose?' The total percentage of respondents 'choosing IS' was $76.8 \%$, 'choosing NIS' was $9.9 \%$, 'both' was $3.9 \%$ and 'does not matter' was $9.3 \%$. The respondents from the capital cities chose IS at a higher rate $(79.5 \%)$ than those from the coastal cities $(72 \%)$. The percentages of respondents choosing NIS was opposite between the city and coastal residents $(6.7 \%$ and $15.7 \%$, respectively), and the ratio of respondents who chose 'both' $(<5 \%)$ and 'does not matter' $(<10 \%)$ were nearly the same in the two areas. Of the 23 cities, 
Figure 1 Flow chart (IDD, iodine deficiency disorder; IS, iodised salt; NIS, non-iodised salt).

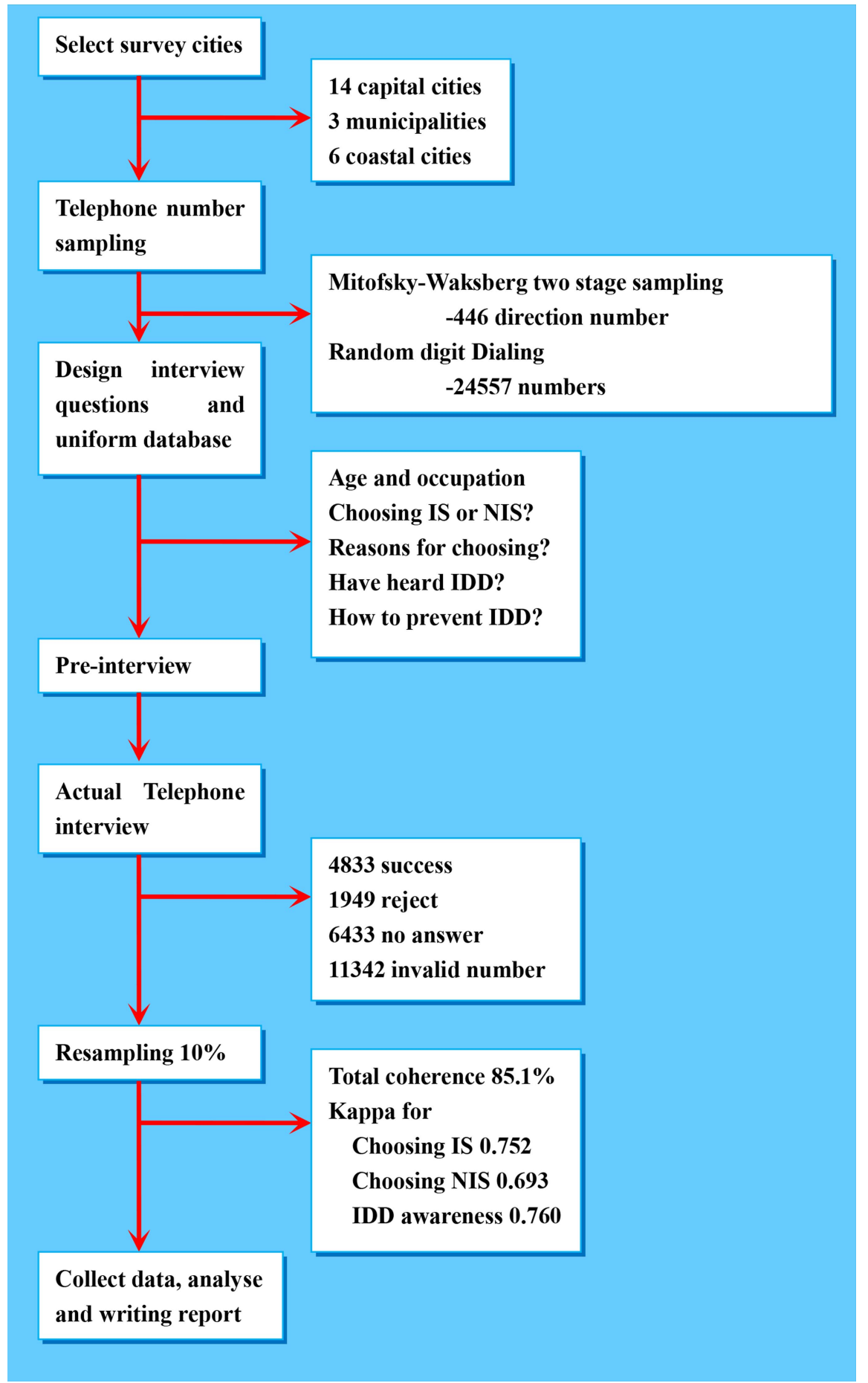

there were 12 cities whose IS selection rate was higher than $80 \%, 8$ cities in which the IS selection rate was between $60 \%$ and $80 \%$, and 3 cities (Shanghai: $49.3 \%$, Zhoushan: $42.8 \%$ and Hangzhou: $32.8 \%$ ) in which the IS selection rate was lower than $50 \%$ (tables 2 and 3).

\section{Reasons for 'choosing IS'}

Of the 3738 residents who chose IS, their reasons were as follows: $61.9 \%$ of them accurately understood the benefits of IS, that is, preventing IDD and increasing children's IQ, 14.7\% of them merely chose IS for superior-quality reasons and knew little about the relationship between IS and IDD prevention, and $27.7 \%$ had no clear reason (most explained that it was the custom) (table 2).

\section{Reasons for 'choosing NIS'}

Most of the 481 residents who chose NIS lived in Shanghai, Hangzhou, Zhoushan and Beihai. The majority thought that they had received an adequate amount 


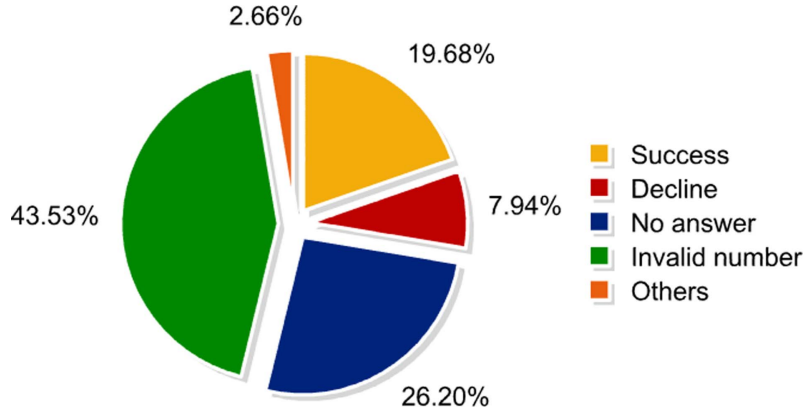

Figure 2 The answer status of the sampled telephone numbers.

of iodine from food or that they did not live in an IDD area. Unexpectedly, $9 \%$ of the respondents who chose NIS thought IS was harmful. Nearly $27 \%$ chose NIS because of reasons that cannot be classified, such as habit, parent's decision or changing taste (table 3).

\section{Reasons for 'choosing both'}

Most of the 192 residents who chose 'both IS and NIS' were from Hangzhou, Guangzhou and Shanghai. The two major reasons were to maintain an appropriate iodine intake and to avoid the harmful effect of excess iodine, accounting for $27.6 \%$ and $30.7 \%$ of the responses, respectively.

\section{Reasons for 'does not matter'}

The 454 residents who chose 'does not matter' mainly resided in Nanjing, Wuhan, Hangzhou, Changsha, Guangzhou, Nanning, Tianjin, Shanghai and Zhoushan. They tended to choose IS or NIS based on which was most convenient to buy ( $52 \%$ of the respondents who had no preference).

\section{Awareness rates of IDD}

Of the residents who responded to our phone calls, 4865 answered the question "Have you heard of IDD?". Among those, 68.7\% (3344) had heard and 31.3\% (1521) had never heard of IDD. There was little difference in having heard of IDD between the capital cities $(68.2 \%)$ and the coastal cities $(69.8 \%)$. The awareness rate in Yantai $(94.5 \%)$ was the highest, and in four cities (Shijiazhuang, Jinan, Hangzhou and Tangshan), the awareness rates were greater than $80 \%$. Beihai and Taiyuan had the lowest awareness rates $(46.2 \%$ and $44.7 \%$, respectively). There were no significant differences in the levels of awareness between age groups or occupation groups.

Table 2 Respondents' reasons for buying iodised salt (IS) (\%)

\begin{tabular}{|c|c|c|c|c|c|c|c|}
\hline \multirow[b]{2}{*}{ Province } & \multirow[b]{2}{*}{ City } & \multirow[b]{2}{*}{$\begin{array}{l}\text { Number of } \\
\text { choosing IS }\end{array}$} & \multirow[b]{2}{*}{$\begin{array}{l}\text { IS choosing } \\
\text { rate }\end{array}$} & \multicolumn{4}{|l|}{ Reasons } \\
\hline & & & & Prevent IDD & $\begin{array}{l}\text { Benefit for } \\
\text { children's IQ }\end{array}$ & Good quality & Others \\
\hline Heilongjiang & Harbin & 183 & 91.5 & 36.6 & 3.8 & 1.6 & 60.1 \\
\hline Jilin & Changchun & 175 & 86.6 & 48.6 & 9.7 & 6.3 & 0 \\
\hline Beijing & - & 202 & 86.7 & 61.4 & 12.9 & 9.4 & 28.2 \\
\hline Hebei & Shijiazhuang & 174 & 87.0 & 67.8 & 7.5 & 12.1 & 14.4 \\
\hline Shanxi & Taiyuan & 189 & 95.0 & 13.8 & 0 & 14.3 & 70.9 \\
\hline Shandong & Ji'nan & 187 & 93.5 & 73.3 & 27.3 & 20.3 & 11.2 \\
\hline He'nan & Zhengzhou & 177 & 88.5 & 54.8 & 15.3 & 26.6 & 27.7 \\
\hline Jiangsu & Nanjing & 180 & 72.6 & 64.4 & 25.0 & 23.9 & 7.8 \\
\hline Anhui & Hefei & 178 & 84.8 & 32.0 & 0.6 & 5.6 & 61.8 \\
\hline Hubei & Wuhan & 163 & 75.1 & 41.7 & 8.0 & 24.5 & 29.4 \\
\hline Zhejiang & Hangzhou & 66 & 32.8 & 57.6 & 16.7 & 21.2 & 0 \\
\hline Jiangxi & Nanchang & 184 & 86.8 & 52.2 & 7.6 & 13.6 & 8.7 \\
\hline Hu'nan & Changsha & 146 & 72.3 & 56.2 & 6.8 & 5.5 & 39.7 \\
\hline Guangdong & Guangzhou & 124 & 61.7 & 39.5 & 1.6 & 46.0 & 3.2 \\
\hline Guangxi & Nanning & 170 & 78.0 & 38.8 & 4.1 & 6.5 & 46.5 \\
\hline \multicolumn{2}{|c|}{ Capital city total } & 2498 & 79.5 & 79.5 & 9.8 & 15.0 & 29.0 \\
\hline Hebei & Tangshan & 208 & 94.1 & 81.7 & 17.3 & 9.6 & 5.8 \\
\hline Tianjin & - & 200 & 83.7 & 75.5 & 28.5 & 27.0 & 6.5 \\
\hline Shandong & Yantai & 186 & 93.0 & 90.3 & 3.2 & 8.1 & 4.8 \\
\hline Jiangsu & Nantong & 153 & 73.9 & 46.4 & 5.9 & 2.6 & 47.7 \\
\hline Shanghai & - & 108 & 49.3 & 25.9 & 6.5 & 29.6 & 48.1 \\
\hline Zhejiang & Zhoushan & 86 & 42.8 & 68.6 & 12.8 & 18.6 & 0 \\
\hline Guangdong & Shenzhen & 144 & 71.6 & 22.9 & 0.7 & 3.5 & 42.4 \\
\hline Guangxi & Beihai & 155 & 66.2 & 21.9 & 2.6 & 18.7 & 58.1 \\
\hline \multicolumn{2}{|c|}{ Coastal city total } & 1240 & 72.0 & 72.0 & 10.6 & 14.1 & 25.0 \\
\hline \multicolumn{2}{|l|}{ Total } & 3738 & 76.8 & 51.9 & 10.0 & 14.7 & 27.7 \\
\hline
\end{tabular}


Table 3 Respondents' reasons for choosing NIS (\%)

\begin{tabular}{llclllllll}
\hline \multicolumn{7}{c}{ Reasons } \\
\cline { 3 - 9 } City & $\mathbf{n}$ & Percent & $\begin{array}{l}\text { lodine } \\
\text { sufficient }\end{array}$ & $\begin{array}{l}\text { Not IDD } \\
\text { endemia }\end{array}$ & Patient* & IS is harmful & IS bad taste & Cheap & Others \\
\hline Capital city Total & 211 & 6.7 & 40.0 & 13.3 & 9.0 & 10.5 & 2.9 & 1.4 & 23.3 \\
Coastal city Total & 270 & 15.7 & 27.7 & 17.7 & 5.2 & 8.1 & 4.0 & 1.5 & 29.9 \\
Total & 481 & 9.9 & 33.1 & 16.6 & 7.3 & 9.1 & 3.5 & 1.5 & 27.0 \\
\hline
\end{tabular}

*Patients with thyroid disease for whom it is not feasible to take IS.

Some provinces had small sample sizes; the proportions refer to large ones; n: number that chose NIS; \%: NIS choosing rate, the reason for choosing NIS was a multiple choice question. IDD, iodine deficiency disorder; IS, lodised salt; NIS, non-iodised salt.

Among the 3344 residents who had heard of IDD, up to $77.1 \%$ of them knew that iodine deficiency could lead to goitre, but only $11.8 \%$ knew that it could also cause intelligence damage to children, and $16.9 \%$ of them knew nothing about its hazard.

\section{Awareness rates of IDD preventive measures}

Of the 3344 residents who had heard of IDD, $62.5 \%$ of them knew IS could prevent IDD, $43.2 \%$ of them knew that kelp and purple seaweed could prevent IDD, and nearly $18.7 \%$ of the residents knew nothing about how to prevent IDD.

\section{The IS preference rates and IDD awareness rates in each city}

All cities could be divided into four groups according to their relationship between the IS preference rate and IDD awareness rate: (1) high IS preference rate and high IDD awareness rate, such as Yantai, Shijiazhuang, Beijing, Tianjin, Nanjing, Changsha and Guangzhou; (2) high IS preference rate and low IDD awareness rate, such as Taiyuan, Harbin, Nanchang, Hefei, Wuhan and Beihai; (3) approximately or equal to the choosing rate and the awareness rate, such as Tangshan, Ji'nan, Zhengzhou, Changchun, Nanning, Nantong and Shenzhen and (4) low IS choosing rate and high IDD awareness rate, such as Shanghai, Zhoushan and Hangzhou. Only in Yantai and Nanjing were the IDD awareness rates a little higher than the IS preference rates. The IS choosing rates in Harbin and Taiyuan were greater than $90 \%$, but their IDD awareness rates were only $57 \%$ and $44.7 \%$, respectively. There were only four cities whose IS rates and awareness rates were all greater than $80 \%$ (table 4 ).

The $\chi^{2}$ test was used to analyse the influence of IDD awareness on the IS preference rate. The results showed no difference between the capital cities' IS and NIS groups but significant difference between the coastal cities' IS and NIS groups. This could explain the weak relations between the IS preference rates and the IDD awareness rates in the cities, whereas for coastal cities, there were associations between the IS preferences and IDD awareness; specifically, part coastal residents who chose NIS had lower IDD awareness rates.

\section{Results of the return visits}

The total coherence rate of all questions was $85.1 \%$. The $\kappa$ values of the coherence analysis for choosing IS, choosing NIS and IDD awareness were 0.752, 0.693 and 0.76 , respectively, and were all above the required 0.4 , describing good coherence. ${ }^{15}$

\section{DISCUSSIONS}

Telephone interview has the advantages. ${ }^{16}$ However, if the response rate $<30 \%,{ }^{17}$ the representation of the random samples will be questioned. In our study, the response rate was $71.3 \%$, and the coherence rate of resurvey was above $80 \%$. One limitation was that it was the first telephone survey on the KAP towards IDD in China; the traditional Pencil-And-Paper-Interview (PAPI) method was taken rather than Computer-AidedTelephone Interview (CATI). The other, some 'other reason' should be recorded more carefully.

Table 4 The iodine deficiency disorder (IDD) awareness rates and iodised salt (IS) preference rates (\%)

\begin{tabular}{lll}
\hline City & IS rate (\%) & $\begin{array}{l}\text { Acknowledge } \\
\text { rate (\%) }\end{array}$ \\
\hline Taiyuan & 95.0 & 44.7 \\
Tangshan & 94.1 & 85.1 \\
Jinan & 93.5 & 81.0 \\
Yantai & 93.0 & 94.5 \\
Harbin & 91.5 & 57.0 \\
Zhengzhou & 88.5 & 74.0 \\
Shijiazhuang & 87.0 & 85.0 \\
Nanchang & 86.8 & 67.5 \\
Beijing & 86.7 & 79.8 \\
Changchun & 86.6 & 65.8 \\
Hefei & 84.8 & 52.9 \\
Tianjin & 83.7 & 76.6 \\
Nanning & 78.0 & 63.8 \\
Wuhan & 75.1 & 59.0 \\
Nantong & 73.9 & 62.8 \\
Nanjing & 72.6 & 74.2 \\
Changsha & 72.3 & 69.8 \\
Shenzhen & 71.6 & 60.7 \\
Beihai & 66.2 & 46.2 \\
Guangzhou & 61.7 & 65.2 \\
Shanghai & 49.3 & 57.5 \\
Zhoushan & 42.8 & 77.6 \\
Hangzhou & 32.8 & 81.1 \\
\hline
\end{tabular}


The 6 coastal cities and 14 capital cities in 14 provinces and 3 municipalities had IDD elimination for nearly 10 years. A phenomenon that emerged in this study was that most residents had lower IDD awareness rates than IS selection rates, as was observed in Taiyuan, Hefei, Harbin and Changchun. Although IDD health education activities, such as the 5.15 IDD day, have been carried out widely and continuously by the relevant departments, it is still not enough or exhaustive, and the current propaganda strength and methods need further improving.

Considering that the supply system of IS is divided by province in China, the KAP towards IDD of the city residents is more important. Although these provinces all attained the goal of IDD elimination and their capital cities and coastal cities are all developed cities, their IS choosing and IDD awareness rates were different, and they should be treated differently according to these results. The result shows that the IS preference rates of southern city residents were lower than those of northern city residents. The main reason was that the people in the southern coastal areas think that they have received adequate iodine from food other than IS, and another reason was that they thought they did not live in an iodine-deficient area, as was the case with the residents of Hangzhou, Zhoushan and Shanghai. Their IDD awareness rates were greater than their IS preference rates, and some residents chose NIS despite knowing about the potential damage caused by IDD. In fact, these districts had no apparent IDD prevalence, which is likely attributable to the widespread IS supply. The survey "Iodine nutritional status of coastal districts residents in 2009" conducted by the $\mathrm{MOH}^{18}$ found that under the condition of consumption rates of qualified IS greater than $90 \%$, the median urinary iodine (MUI) of Shanghai residents was merely between 100 and $200 \mu \mathrm{g} / \mathrm{L}$, that is, just at the iodine intake appropriate level, and the MUI of pregnant women was lower than $150 \mu \mathrm{g} / \mathrm{L}$, which corresponds to an insufficient iodine intake level. Among the residents of the Xiacheng district of Hangzhou city, Zhejiang province, the consumption rate of qualified IS was $96.7 \%$, and the MUI of 8-10-year-old children was between 100 and $200 \mu \mathrm{g} / \mathrm{L}$, which is at the iodine intake appropriate level. ${ }^{18}$ If allowing these cities' residents to freely choose IS or NIS, the residents may choose NIS blindly, and the achievement of controlled IDD that has been obtained will be destroyed.

From the society and economic development aspects, supplying IS and NIS simultaneously to some cities or persons is an inevitable trend. In the public health domain, if a disease has a prevalence rate below $5 \%$, it will not become a public health problem. Hence, we deem that if the IDD awareness rates and the correct selection rates are above $95 \%$, IS and NIS can both be made available. According to this telephone interview study, it is not a suitable time to supply IS and NIS simultaneously, even in the developed cities of China. The intake of NIS should be performed with caution in China. The next step is to conduct a trial in which IS and NIS is simultaneously supplied in appropriate cities. The selected experimental city should be a developed city with no IDD prevalence in recent years, and the residents' awareness rate of IDD and selection rate of IS should be high. According to our study, Yantai, Tangshan and Ji'nan may be appropriate cities for this experiment. Before the experiment, emergency planning should be performed, and extensive IDD education should be conducted. If abnormal situations emerge, the emergency programme will be started, or the trial will even be stopped. Currently, USI was carried out as a mandatory effective strategy to prevent IDD in China since 1996. However, no documentation was found about the residents' preferences on salt type (IS or NIS) based on their awareness of IDD and its prevention measure knowledge. Hence, the study tried to find out the reason why people choose IS or NIS. Reasons enlisted including preventing IDD, improving children's IQ good quality and convenience, etc, were possible main reasons people would purchase IS, while, the potential harm and having thyroid disorders were the reasons for choosing NIS. These results were important for further research and prevention measures and policy decision.

\section{Author affiliations}

${ }^{1}$ Institute for Kaschin-Beck Disease Control, the Centre for Endemic Disease Control, Harbin Medical University, Harbin, People's Republic of China ${ }^{2}$ Institute for lodine Deficiency Disorders, the Centre for Endemic Disease Control, Harbin Medical University, Harbin, People's Republic of China

${ }^{3}$ The Centre for Endemic Disease Control, Harbin Medical University, Harbin, People's Republic of China. Key lab of Etiology and Epidemiology, Education Bureau of Heilongjiang Province \& Ministry of Health, Harbin, China

Acknowledgements The authors thank the organisations that participated in this survey: the National Centre for Endemic Disease Control (CEDC), 16 provincial (municipality) Centres for Disease Control (CDC) or CEDC, including those of the Beijing, Tianjin, Shanghai, and Jilin, Hebei, Shandong, He'nan, Jiangsu, Anhui, Hubei, Zhejiang, Jiangxi, Hu'nan, Guangdong and Guangxi province, and six coastal city CDCs, including those of Tangshan, Yantai, Nantong, Zhoushan, Shenzhen and Beihai. They also thank those colleagues from the above organisations who took part in the survey for their hard work and thank Elsevier English Language Editing for their English editing.

Contributors YJ wrote the project report, LP analysed the data and wrote the manuscript, LY and SL designed the study and managed the project and DS was responsible for the project and the publication.

Funding The project was funded by the Ministry of Health of China (2010zzxm). Competing interests None.

Ethics approval The review board of the Harbin Medical University.

Provenance and peer review Not commissioned; externally peer reviewed.

Data sharing statement No additional data are available.

Open Access This is an Open Access article distributed in accordance with the Creative Commons Attribution Non Commercial (CC BY-NC 4.0) license, which permits others to distribute, remix, adapt, build upon this work noncommercially, and license their derivative works on different terms, provided the original work is properly cited and the use is non-commercial. See: http:// creativecommons.org/licenses/by-nc/4.0/ 


\section{REFERENCES}

1. WHO/UNICEF/ICCIDD. Assessment of iodine deficiency disorders and monitoring their elimination. A guide for programme managers. 3rd edn. France: World Health Organization, 2007.

2. Todd $\mathrm{CH}$, Allain T, Gomo ZA, et al. Increase in thyrotoxicosis associated with iodine supplements in Zimbabwe. Lancet 1995;346:1563-4.

3. Stanbury JB, Ermans AE, Bourdoux $P$, et al. lodine-induced hyperthyroidism: occurrence and epidemiology. Thyroid 1998;8:83-100.

4. Parveen S, Latif SA, Kamal MM, et al. lodized salt induced thyrotoxicosis: Bangladesh perspective, Mymensingh. Med J 2009;18:165-8.

5. ICCIDD. International Council for the Control of lodine Deficiency Disorder. CIDDS Database. Current IDD Status Database, 2004

6. Groves RM, Biemer PP, Lyberg LE, et al. Telephone survey methodology. John Wiley \&Sons Inc, 1989, 2-10.

7. Jianhua L, Hanwu M, Yongsheng W, et al. Analysis of knowledge, attitude and practice of the influenza $\mathrm{A} / \mathrm{H} 1 \mathrm{~N} 1$ of general and immigration population in Shenzhen. Chin Health Educ 2009;25:915-8.

8. Jin M, Shunxiang Z, Hanwu M, et al. Telephone survey on behavior risk factors of Shenzhen residents among six districts in 200. J Dis Control 2005;9:561-5.
9. Tao W, Su-jun L, Liang Z, et al. Investigation of pollinosis in Beijing residents over the age of 15. J Environ Health 2008;25:403-4.

10. Blair G. Obstetricians' receptiveness to teen prenatal patients who are medicaid recipients. Health Serv Res 1997;32: 265-82.

11. National Bureau of Statistics of China. Statistics Bulletin of the Cities' Economic and Social Development of 2009.

12. Potthoff RF. Some Generazations of the Mitofsky-Waksberg techniques for random digital dialing. J Am Stat Assoc 1987;82:409-41.

13. Yi MING. http://geo.cersp.com/sJxzy/sc/200706/2658.html

14. http://www.moh.gov.cn/publicfiles/business/htmlfiles/zwgkzt/ptinj/ 200908/42635.htm

15. Jiezhen $\mathrm{W}$, Jing $\mathrm{H}$, Yanxun $\mathrm{L}$, et al. Kappa statistic value and its application. Chin Health Stat 1995;12:46-50.

16. Ping W, Weijing D. Telephone survey and its application in public health. Health Educ China 2004;20:549-50.

17. Guohua D. A powerful tool of the statistic survey-the computer aid telephone survey system. Newspaper Theory Guidance 2008;12:51-2.

18. Chinese CDC, CEDC. Report of coastal residents' iodine nutrient level. 2009. 